\title{
Initial experience with endoscopic ultrasound-guided gallbladder drainage
}

\author{
Sebastian Sowier ${ }^{1}$, Aleksander Sowier ${ }^{1}$, Anna Wiechowska-Kozłowska², Jacek Białecki ${ }^{1}$, Przemysław Pyda ${ }^{1,3}$ \\ ${ }^{1}$ Department of General, Minimally Invasive and Trauma Surgery, Franciszek Raszeja City Hospital, Poznan, Poland \\ ${ }^{2}$ Department of Internal Medicine, Hospital of the Ministry of Interior and Administration, Szczecin, Poland \\ ${ }^{3}$ Department of General and Endocrine Surgery and Gastroenterological Oncology, Poznan University of Medical Sciences, Poznan, Poland \\ Videosurgery Miniinv 2019; 14 (2): 195-202 \\ DOI: https://doi.org/10.5114/wiitm.2018.79528
}

\begin{abstract}
Introduction: Patients with acute cholecystitis who are unsuitable for cholecystectomy undergo conservative treatment or percutaneous transhepatic gallbladder drainage. As these two methods are not always successful, further treatment options are needed. One increasingly popular method is endoscopic ultrasound-guided gallbladder drainage (EUSGBD), whereby stents are placed so as to create a permanent fistula connecting the gallbladder to the stomach or the duodenal bulb, thus enabling drainage of its contents to the gastrointestinal tract.

Aim: To present our early experience with EUSGBD for the treatment of cholecystitis in patients who are not suitable for cholecystectomy.

Material and methods: The procedure was performed in 5 patients with acute cholecystitis. Two patients also had symptoms of biliary obstruction due to pancreatic head cancer. An ultrasound endoscope was used to create a fistula between the gallbladder and the stomach or between the gallbladder and the duodenal bulb, in which a self-expandable metallic stent (SEMS) was placed.

Results: All procedures were performed with no perioperative complications. In all patients, the clinical symptoms of cholecystitis subsided within 3-15 days. In those patients who also showed symptoms of biliary obstruction, these symptoms subsided within 3-6 days following the procedure.

Conclusions: The EUSGBD seems to be an effective and safe treatment for acute cholecystitis in patients unsuitable for cholecystectomy. It can also be used to treat jaundice caused by obstruction of the common bile duct, when no other methods can be used. The method is particularly promising in cases of concurrent acute cholecystitis and common bile duct obstruction.
\end{abstract}

Key words: cholecystectomy, acute cholecystitis, EUS-guided gallbladder drainage, obstructive jaundice.

\section{Introduction}

Cholecystectomy is the standard treatment for acute cholecystitis once conservative treatment has proven ineffective. Unfortunately not all patients are suitable for this procedure. The most common contraindications for cholecystectomy are: old age, severe comorbidities, especially cancer, and previous abdominal surgery [1-3]. In patients with advanced cancer located in the area of the hepatic hilum or pancreas, or patients who have undergone previous surgery in this area, cholecystectomy may turn out to be much more difficult to perform and may be associated with more complications than the standard procedure. In those patients (especially when they are in poor overall condition) there is a need for a different, less invasive mode of therapy. Until now the only alternative for cholecystectomy in such cas-

\section{Address for correspondence}

Sebastian Sowier, Department of General, Minimally Invasive and Trauma Surgery, Franciszek Raszeja City Hospital, 2 Mickiewicza St, 60-834 Poznan, Poland, e-mail: ssowier@gmail.com 
es (besides conservative treatment) has been percutaneous transhepatic gallbladder drainage (PTGBD) [4-6]. However, PTGBD involves a risk of multiple complications, including biliary peritonitis, bleeding, catheter migration, dislodgement, or obstruction, pneumothorax, and bile leak [7-9]. Practical difficulties associated with the percutaneous catheter are also a considerable disadvantage of PTGBD, as the catheter has to be checked for dislocation or obstruction, and the patient needs to remain in a forced position while resting, and may experience pain. Percutaneous drainage also results in dehydration and electrolyte imbalance. Moreover, it may be difficult to decide on the right time to discontinue treatment. Additionally, PTGBD may be difficult or contraindicated in patients with ascites, coagulopathy, or dementia $[10,11]$. These factors necessitate a search for new treatments in patients unfit for cholecystectomy. Recently, endoscopic ultrasound-guided gallbladder drainage (EUSGBD) has been gaining popularity [12-14]. The EUSGBD method consists in stent placement so as to create a permanent fistula connecting the gallbladder to the stomach or the duodenal bulb, thus enabling drainage of its contents to the gastrointestinal tract. Biliary drainage to the gastrointestinal tract, rather than percutaneously to the outside of the body, enhances patient comfort due to the lack of a percutaneous catheter. Bile is drained from the gallbladder in a more physiological manner, preventing the loss of all its components secreted by the liver. The EUSGBD is still a new procedure, and the discussion regarding the proper technique and equipment is still ongoing, involving such issues as the location of the fistula, the type of

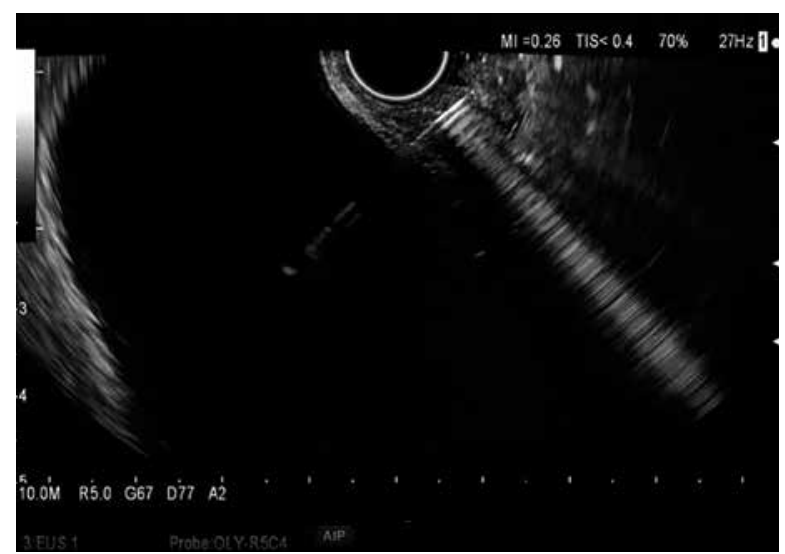

Photo 1. Gallbladder puncture with a 19-gauge needle stents to be used and the appropriate time for stent removal. There is also the question about the possibility of using EUSGBD as an alternative to cholecystectomy in cases of cholecystitis and cholelithiasis. Beside the treatment of cholecystitis, other applications for EUSGBD also exist [15]. The method may be used for treatment of obstructive jaundice due to common bile duct (CBD) obstruction, when currently used methods such as bile duct dilation or stenting (endoscopic or percutaneous), or endoscopic ultrasound-guided biliary drainage, cannot be used or have proven ineffective. The formed fistula can also be used for performing cholecystoscopy with the removal of concretions or polyps.

\section{Aim}

The purpose of the paper was to present our early experience with EUS-guided gallbladder drainage in the treatment of cholecystitis in patients who are not suitable for cholecystectomy.

\section{Material and methods}

The procedure was performed in 5 patients ( $1 \mathrm{fe}$ male) aged 27-81 years (mean age: 67 years) with acute cholecystitis. Two of the patients also had symptoms of jaundice due to obstruction of the CBD in the course of pancreatic head cancer. In 4 patients, cholecystectomy was contraindicated due to advanced cancer, and in one, cardiovascular and neurological contraindications existed. The procedures were performed under general anesthesia. A $14.6 \mathrm{~mm}$ single channel ultrasound endoscope with a $3.7 \mathrm{~mm}$ inner diameter (Olympus, Tokyo, Japan) was inserted into the duodenum, to identify the gallbladder and locate it relative to the gastrointestinal wall. Anatomy may differ between patients - in some, a larger portion of the gallbladder wall may be adjacent to the pyloric part of the stomach or to the duodenal bulb than in others. Given these differences, we performed a cholecystogastric anastomosis in 2 cases, and a cholecystoduodenal anastomosis in the 3 remaining ones. After determining the fistula location, the gallbladder was punctured with a 19-gauge needle introduced through the endoscope channel (Photo 1), and bile aspiration was performed to verify the correct identification of the gallbladder. When selecting the puncture location, we also assessed the locations of major blood vessels using Doppler imaging. A 0.035 inch guidewire 
(Boston Scientific Dreamwire) was advanced through the needle inserted in the gastric or duodenal wall. The guidewire was coiled to prevent dislodgement at subsequent stages of the procedure. The needle was withdrawn, and an 8.5 Fr cystotome (Cook Medical, Bloomington, Indiana) was introduced over the guidewire. The fistula between the gastric or duodenal wall and the gallbladder wall was enlarged by electrocoagulation. Once the fistula had been formed, the cystotome was withdrawn, and a fully covered self-expandable metallic stent (SEMS) $4 \mathrm{~cm}$ in length and $1 \mathrm{~cm}$ in diameter was advanced over the guidewire using a special delivery system (Boston Scientific WallFlex). The delivery system consists of an exterior tube, metal stent and a delivery catheter. After the exterior tube was removed, the stent fully expanded, creating the anastomosis. Next the delivery catheter was removed along with the guidewire. To avoid bile leakage, we decided against gallbladder lavage. Additionally, a $4 \mathrm{~cm}$ 8.5 Fr double pigtail stent was placed in the SEMS lumen to secure the anastomosis and prevent SEMS migration (Photo 2). The mean operation time was $33 \mathrm{~min}$. In one patient, the SEMS was removed 30 days after the procedure, while in the remaining patients, the SEMS were not removed.

\section{Results}

All the procedures were performed with no perioperative complications. In all patients, the clinical symptoms of cholecystitis subsided within 3-15 days (mean: 8.6 days). During this time, signs of inflammation, such as increased C-reactive protein (CRP) levels, increased white blood cells count (WBC), and fever, decreased considerably (Table I). In the 2 patients showing symptoms of CBD obstruction in addition to cholecystitis, these symptoms subsided within 3-6 days following the surgery (mean: 4.5 days), and a significant decrease of bilirubin levels was observed (Table I). Four weeks after the procedure, the patients were admitted for a 1-day follow-up hospitalization, including a physical examination, ultrasound examination, gastroscopy, and laboratory tests (4 patients were followed up, as 1 patient died due to cancer progression). Cholecystitis did not recur in any of the 4 patients. The patients reported no pain, either fasting or postprandial, and had no fever. In 3 patients, laboratory inflammation markers were normal; 1 patient had an

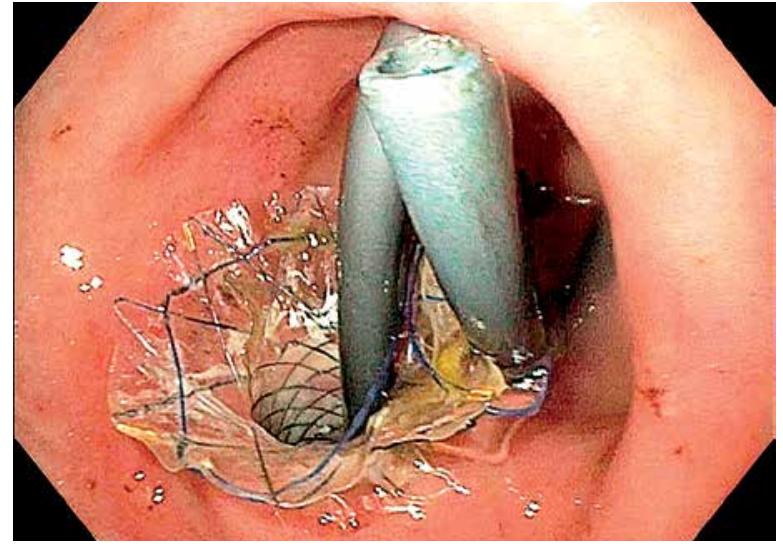

Photo 2. Double pigtail stent placed in the SEMS lumen to prevent SEMS migration

increased CRP level (Table I). In 3 patients, gastroscopy showed unobstructed, functional anastomoses; therefore, both the SEMS and the pigtail stents were left in place to continue drainage. In 1 of these patients, the fistula was used to remove a gallbladder polyp. In the other 2 of these patients, the examination showed biliary concretions, which were also removed through the fistula. In the remaining patient, the follow-up showed that the SEMS had migrated to the gallbladder lumen, and the cholecystoduodenal fistula had been obliterated. The SEMS migration into the gallbladder was also confirmed by abdominal ultrasound examination. The pig-tail stent could not be located - it had likely moved to the lumen of the gastrointestinal tract, loosening the SEMS and enabling its migration. We continued monitoring the patient's condition, and another evaluation for cholecystectomy was performed after 3 months, but the procedure was not performed due to cardiovascular contraindications. During subsequent follow-ups, at 6 and 12 months, the patient had no symptoms of cholecystitis or any other complaints, despite the SEMS in the gallbladder lumen.

\section{Discussion}

Cholecystectomy is a common, relatively safe procedure, with a low complication rate, and clearly defined, commonly accepted indications. In some situations, however, the patient's condition does not allow for the procedure to be performed. Patients with cholecystitis in whom conservative treatment has not been effective and who are not suitable for cholecystectomy may be treated with PTGBD. This method, however, has multiple disad- 
Table I. Surgical treatment outcomes

\begin{tabular}{|c|c|c|c|c|c|c|}
\hline \multirow[t]{2}{*}{ Parameter } & \multicolumn{5}{|c|}{ Patient } & \multirow[t]{2}{*}{ Mean } \\
\hline & 1 & II & III & IV & $\mathrm{V}$ & \\
\hline Age [years] & 73 & 81 & 27 & 75 & 80 & 67 \\
\hline $\begin{array}{l}\text { Diagnosis ( } a \text { - acute cholecystitis, } b \text { - common bile } \\
\text { duct obstruction) }\end{array}$ & a & $a, b$ & a & $a, b$ & a & - \\
\hline $\begin{array}{l}\text { Comorbidities (a - pancreatic head cancer, } \\
b \text { - cholelithiasis) }\end{array}$ & a & a & a & a & $b$ & - \\
\hline Anastomosis location (a - stomach, b- duodenal bulb) & a & $b$ & a & $b$ & $b$ & \\
\hline Time of hospitalization in days & 10 & 6 & 9 & 3 & 15 & 8.6 \\
\hline \multicolumn{7}{|l|}{ Parameters before the procedure: } \\
\hline $\mathrm{CRP}[\mathrm{mg} / \mathrm{dl}]$ & 80 & 152 & 333 & 61 & 314 & 188 \\
\hline Serum WBC $\left[10^{3} / \mu l\right]$ & 16 & 11 & 13 & 10 & 16 & 13.2 \\
\hline Bilirubin [mg/dl] & - & 8 & - & 20 & - & 14 \\
\hline Fever & Yes & Yes & Yes & No & Yes & - \\
\hline Complications & No & No & No & No & No & - \\
\hline \multicolumn{7}{|l|}{ Parameters at discharge: } \\
\hline $\mathrm{CRP}[\mathrm{mg} / \mathrm{dl}]$ & 20 & 40 & 22 & 42 & 73 & 39.4 \\
\hline Serum WBC $\left[10^{3} / \mu \mathrm{l}\right]$ & 8 & 10 & 8 & 6 & 8 & 8 \\
\hline Bilirubin [mg/dl] & - & 3 & - & 4 & - & 3.5 \\
\hline Fever & No & No & No & No & No & - \\
\hline Complications & No & No & No & No & No & - \\
\hline \multicolumn{7}{|l|}{ Parameters 4 weeks after discharge ${ }^{1}$ : } \\
\hline $\mathrm{CRP}[\mathrm{mg} / \mathrm{dl}]$ & 2 & - & 4 & 30 & 4 & 10 \\
\hline Serum WBC $\left[10^{3} / \mu l\right]$ & 6 & - & 10 & 6 & 6 & 7 \\
\hline Bilirubin [mg/dl] & 0.4 & - & 0.4 & 2.5 & 0.6 & 0.975 \\
\hline Fever & No & - & No & No & No & - \\
\hline Complications & No & - & No & No & $\begin{array}{l}\text { Stent } \\
\text { migration }\end{array}$ & - \\
\hline
\end{tabular}

vantages, and the use of EUSGBD in lieu of PTGBD is considered increasingly often. Despite numerous references in the literature EUSGBD is still performed rather seldom in Poland. This may change due to the increasing access to endoscopic procedures. One might ask whether EUSGBD can be regarded as a less invasive procedure compared to laparoscopic cholecystectomy, since EUSGBD is also performed under general anesthesia. The EUSGBD is a significantly shorter procedure (mean operation time: $33 \mathrm{~min}$ ), especially when compared with lap- aroscopic cholecystectomy performed in patients with tumors located in the hepatic hilum or the head of the pancreas. In such cases one may expect serious difficulties and a high probability of converting to laparotomy. This may lengthen the procedure several times. Additionally, EUSGBD does not require creation of pneumoperitoneum, and does not result in postoperative wounds, which helps to avoid numerous complications. A number of publications compare EUSGBD and PTGBD [14]. Tyberg et al. report that the two methods do not significant- 
ly differ in terms of technical and clinical success or complication rate, but that EUSGBD is associated with a lower percentage of re-intervention [16]. Irani et al. found similar rates of technical and clinical success for both procedures, but indicated that patients treated with EUSGBD required shorter hospitalization, reported less pain, required fewer re-interventions, and had a slightly lower complication rate [17]. Ahmed et al. performed a meta-analysis of 5 studies comparing PTGBD against EUSGBD, reporting similar rates of technical and clinical success, as well as similar mortality associated with both procedures, but concluding that EUSGBD was superior due to fewer complications, less pain, and shorter hospitalization. Furthermore, EUSGBD patients had lower rates of acute cholecystitis recurrence and rehospitalization, though these differences were not statistically significant [18]. These reports suggest that EUSGBD may soon replace PTGBD as a cholecystitis treatment method for patients unsuitable for cholecystectomy who have not been successfully treated conservatively. One may also wonder if EUSGBD could become a fully recognized form of relatively non-invasive treatment for acute cholecystitis. Yeung et al. suggest that in the future EUSGBD might in fact become the standard treatment for gallstones [19].

In cases of bile duct obstruction, the treatment of choice is bile duct stenting via endoscopic retrograde cholangiopancreatography (ERCP). If the ampulla of Vater or the bile duct cannot be accessed, percutaneous transhepatic biliary drainage (PTBD) is performed, but currently, endoscopic ultrasound-guided biliary drainage (EUSBD) is also used in a number of centers [20-22]. As demonstrated by Imai et al., in cases where bile duct stenting via ERCP is not possible due to lack of access, while EUSBD would be difficult due to factors such as duodenal stenosis, bile duct wall thickening, interposing blood vessels, or non-dilated intrahepatic bile ducts, the use of EUSGBD instead of PTBD may be considered (as the patient might want to avoid having a percutaneous catheter placed), provided that the cystic duct is unobstructed [23].

In the present study, in the case of 2 patients in whom symptoms of CBD obstruction (high bilirubin, bile duct dilatation) were found beside cholecystitis, gallbladder drainage also proved effective in draining the bile ducts. Though attempts to treat CBD obstruction with EUSGBD are only warranted if stenting via ERCP or EUSBD cannot be performed, EUSGBD might turn out to be the preferred treatment in cases where the obstruction co-occurs with cholecystitis and cholecystectomy is contraindicated. Ogura et al. report having successfully treated a patient with acute cholecystitis and CBD obstruction caused by an advanced cancer of the bile duct, using endoscopic ultrasound-guided hepaticogastrostomy (a variant of EUSBD) and EUSGBD [24]. First, hepaticogastrostomy was performed to treat the obstruction, and cholecystitis was subsequently treated with PTGBD. As cholecystitis recurred, the team decided to perform EUSGBD. Based on our experience, in a case of CBD obstruction, performing EUSGBD first could help avoid both PTGBD and EUSHG. Therefore, in some specific cases, EUSGBD might prove to be a comprehensive treatment for acute cholecystitis with concurrent bile duct obstruction in patients unsuitable for cholecystectomy.

Another potential benefit of EUSGBD is also worth attention. As reported by Chan et al., the fistula created during the procedure may be used for performing cholecystoscopy, offering further diagnostic and treatment options for gallbladder diseases (e.g. performing mucosal biopsies or visualizing and removing polyps) [25]. In the present study, the fistula was used to perform polypectomy in 1 patient, and to remove biliary concretions in 2 other patients.

One significant issue in the context of EUSGBD is stent selection. Initially, plastic double pigtail stents were used, though due to their small diameter and small lateral openings, drainage was limited to the liquid gallbladder contents. The use of these stents has nearly ceased, and most centers use either self-expanding metal stents [26] or lumen-apposing metal stents (LAMS) $[27,28]$. The LAMS have been specifically designed for establishing fistulae between organs [29]. Due to their flanged shape, the stents are capable of fixing two non-adjoining organs in a position enabling the formation of a permanent anastomosis [30]. Thus, the use of LAMS is associated with fewer cases of stent migration or bile leak [31]. This warrants the prediction that LAMS may soon replace SEMS. In terms of stent placement, the innovative single-step technique reduces the number of endoscopic instrument changes required to perform the procedure, resulting in shorter procedure times and lower risk of error [32, 33]. When performing the procedures described here, we only had access to SEMS. The LAMS cost several times more than 
SEMS, which means that SEMS may still be used for economic reasons. A follow-up study by Choi et al. indicates that the use of SEMS produces very good long-term results [34]. The risk of SEMS migration may be curbed, as suggested by Takagi et al. [35], by additionally placing a pigtail stent in the lumen of the SEMS, which we did in all the described cases.

Another consideration, though maybe less significant, is the selection of the method for dilating the initial puncture to form a fistula. The available options include the use of a biliary dilation catheter, a balloon dilator, or a cystotome [23]. In our case, the use of a cystotome proved to be an effective solution for dilating the opening efficiently and without bleeding. More significant considerations include decisions about stent removal or fistula closure. Kamata et al., who used SEMS, suggest removing the stents approximately 4 weeks after the surgery, to eliminate the risk of stent migration into the gallbladder or of stent obstruction by food particles, which might lead to cholecystitis recurrence [26]. The authors indicate that in order to avoid cholecystitis recurrence after SEMS removal, placement of a pigtail stent should be considered. Stent migration into the lumen of the gastrointestinal tract, and its subsequent natural elimination through this route, would not constitute a significant risk for the patient. If, however, the stent migrates into the gallbladder (as was the case in one of the present patients), it could cause a variety of unpredictable complications. Leaving the stent and the open fistula in place might result in cholecystitis recurrence, contributed to by gastric or intestinal content reflux into the lumen of the gallbladder. Walter et al., who used LAMS, left the stents in place for 3 months [36]. The authors report that when stents are left in place for that long, tissue overgrowth may occur, interfering with subsequent stent removal, and therefore, leaving the stent permanently should be considered, as this course of action prevents complications and patient discomfort associated with reoperation. A similar view is offered by Choi et al., who reported that no serious complications occurred even when stents remained in place for 3 years [34]. Bearing in mind these recommendations, as well as the fact that 4 out of 5 of our patients had pancreatic cancer, and therefore had a short life expectancy, we decided against stent removal. This is also recommended by Chan and Teoh in patients with a short life expectancy [12].
Another problem is the selection of a location for the fistula, which may be formed between the gallbladder and the stomach, or between the gallbladder and the duodenal bulb. Walter et al. suggest that a more stable anastomosis is possible in the duodenum, due to the better and more stable fixation of the organ itself. The gastric wall can move relative to the adjacent organs, and peristalsis is stronger within the stomach [36]. A gastric anastomosis may also allow food particles to enter the gallbladder.

Takagi et al., who used SEMS, state that a gastric anastomosis is technically easier, but is associated with a higher risk of bile leakage and stent migration, while a duodenal anastomosis is more difficult to perform, but produces better outcomes [35]. Considerations related to the fistula location also include the location of gallbladder puncture. Takagi et al. state that in the case of duodenal access, the neck of the gallbladder should be punctured (as it is less mobile), which prevents stent migration [35]. We attempted to perform a duodenal anastomosis whenever possible, but in 2 out of 5 cases, we performed a gastric anastomosis, as the distance between the gallbladder wall and the duodenal wall was too large, which excluded safe placement of a SEMS.

Since the present study was a retrospective one, and EUSGBD was chosen as the most suitable modality in the given clinical situations, approval from a bioethics committee was not sought.

\section{Conclusions}

The EUSGBD seems to be a feasible, effective and safe treatment for acute cholecystitis in patients not suitable for cholecystectomy, who could not be successfully treated conservatively. Several ways of performing the procedure exist, and their relative effectiveness has not yet been sufficiently examined. Further studies are warranted to determine indications for this method, its efficacy and the optimal method for establishing such drainage. In the future, it is likely that EUSGBD will not only replace PTGBD, but may also become a fully recognized method for the treatment of gallbladder diseases in specific clinical situations. The EUSGBD may also be used to help patients with CBD obstruction in cases where other treatments are not possible. The method is particularly promising in cases of concurrent acute cholecystitis and CBD obstruction. 


\section{Conflict of interest}

\section{The authors declare no conflict of interest.}

\section{References}

1. Keus F, Broeders IA, van Laarhoven CJ. Gallstone disease: surgical aspects of symptomatic cholecystolithiasis and acute cholecystitis. Best Pract Res Clin Gastroenterol 2006; 20: 1031-51.

2. Deziel DJ. Complications of cholecystectomy. Incidence, clinical manifestations, and diagnosis. Surg Clin North Am 1994; 74: 809-23.

3. Mastalerz K, Kenig J, Olszewska U, et al. The Surgical Apgar score and frailty as outcome predictors in short- and long-term evaluation of fit and frail older patients undergoing elective laparoscopic cholecystectomy - a prospective cohort study. Videosurgery Miniinv 2018; 13: 350-7.

4. Kortram K, Vries de Reilingh TS, Wiezer MJ, et al. Percutaneous drainage for acute calculous cholecystitis. Surg Endosc 2011; 25: 3642-6.

5. McGahan J, Lindfors K. Percutaneous cholecystostomy: an alternative to surgical cholecystostomy for acute cholecystitis? Radiology 1989; 173: 481-5.

6. Winbladh A, Gullstrand P, Svanvik J, et al. Systematic review of cholecystostomy as a treatment option in acute cholecystitis. HPB (Oxford) 2009; 11: 183-93.

7. Itoi T, Coelho-Prabhu N, Baron TH. Endoscopic gallbladder drainage for management of acute cholecystitis. Gastrointest Endosc 2010; 71: 1038-45.

8. Hatzidakis AA, Prassopoulos P, Petinarakis I, et al. Acute cholecystitis in high-risk patients: percutaneous cholecystostomy vs conservative treatment. Eur Radiol 2002; 12: 1778-84.

9. Ha JP, Tsui KK, Tang CN, et al. Cholecystectomy or not after percutaneous cholecystostomy for acute calculous cholecystitis in high-risk patients. Hepatogastroenterology 2008; 55: 1497-502.

10. Hasan MK, Itoi T, Varadarajulu S. Endoscopic management of acute cholecystitis. Gastrointest Endosc Clin N Am 2013; 23: 453-9.

11. Itoi T, Sofuni A, Itokawa F, et al. Endoscopic transpapillary gallbladder drainage in patients with acute cholecystitis in whom percutaneous transhepatic approach is contraindicated or anatomically impossible. Gastrointest Endosc 2008; 68: 455-60.

12. Chan JHY, Teoh AYB. Current status of endoscopic gallbladder drainage. Clin Endosc 2018; 51: 150-5.

13. Teoh AYB, Serna C, Penas I, et al. Endoscopic ultrasound-guided gallbladder drainage reduces adverse events compared with percutaneous cholecystostomy in patients who are unfit for cholecystectomy. Endoscopy 2017; 49: 130-8.

14. Khan MA, Atiq O, Kubiliun N, et al. Efficacy and safety of endoscopic gallbladder drainage in acute cholecystitis: is it better than percutaneous gallbladder drainage? Gastrointest Endosc 2017; 85: 76-87.

15. Law R, Baron TH. Endoscopic ultrasound-guided gallbladder drainage. Gastrointest Endosc Clin N Am 2018; 28: 187-95.

16. Tyberg A, Saumoy M, Sequeiros EV, et al. EUS-guided versus percutaneous gallbladder drainage: isn't it time to convert? J Clin Gastroenterol 2018; 52: 79-84.
17. Irani S, Ngamruengphong S, Teoh A, et al. Similar efficacies of endoscopic ultrasound gallbladder drainage with a lumen-apposing metal stent versus percutaneous transhepatic gallbladder drainage for acute cholecystitis. Clin Gastroenterol Hepatol 2017; 15: 738-45.

18. Ahmed O, Rogers AC, Bolger JC, et al. Meta-analysis of outcomes of endoscopic ultrasound-guided gallbladder drainage versus percutaneous cholecystostomy for the management of acute cholecystitis. Surg Endosc 2018; 32: 1627-35.

19. Yeung B, Teoh AY. Endoscopic management of gallbladder stones: can we eliminate cholecystectomy? Curr Gastroenterol Rep 2016; 18: 42.

20. Ogura T, Higuchi K. Does endoscopic ultrasound-guided biliary drainage really have clinical impact? World J Gastroenterol 2015; 21: 1049-52.

21. Oh SY, Irani S, Kozarek RA. What are the current and potential future roles for endoscopic ultrasound in the treatment of pancreatic cancer? World J Gastrointest Endosc 2016; 8: 319-29.

22. Baniya R, Upadhaya S, Madala S, et al. Endoscopic ultrasound-guided biliary drainage versus percutaneous transhepatic biliary drainage after failed endoscopic retrograde cholangiopancreatography: a meta-analysis. Clin Exp Gastroenterol 2017; 10: 67-74.

23. Imai H, Kitano M, Omoto S. EUS-guided gallbladder drainage for rescue treatment of malignant distal biliary obstruction after unsuccessful ERCP. Gastroint Endosc 2016; 84: 147-51.

24. Ogura T, Masuda D, Imoto A, et. al. Higuchi EUS-guided gallbladder drainage and hepaticogastrostomy for acute cholecystitis and obstructive jaundice (with video). Endoscopy 2014; 46 (S 01): E75-6.

25. Chan SM, Teoh AYB, Yip HC, et al. Feasibility of per-oral cholecystoscopy and advanced gallbladder interventions after EUS-guided gallbladder stenting (with video). Gastrointest Endosc 2017; 85: 1225-32.

26. Kamata K, Takenaka M, Kitano M, et al. Endoscopic ultrasound-guided gallbladder drainage for acute cholecystitis: long-term outcomes after removal of a self-expandable metal stent. World J Gastroenterol 2017; 23: 661-7.

27. Patil R, Ona Mel A, Papafragkakis C, et al. Endoscopic ultrasound-guided placement of the lumen-apposing self-expandable metallic stent for gallbladder drainage: a promising technique. Ann Gastroenterol 2016; 29: 162-7.

28. Weilert F, Binmoeller KF. Specially designed stents for translumenal drainage. Gastrointest Interv 2015; 4: 40-5.

29. Binmoeller KF, Shah J. A novel lumen-apposing stent for transluminal drainage of nonadherent extraintestinal fluid collections. Endoscopy 2011; 43: 337-42.

30. Serna-Higuera de la C, Pérez-Miranda M, Gil-Simón P, et al. EUS-guided transenteric gallbladder drainage with a new fistula-forming, lumen-apposing metal stent. Gastrointest Endosc 2013; 77: 303-8.

31. Anderloni A, Buda A, Vieceli F, et al. Endoscopic ultrasound-guided transmural stenting for gallbladder drainage in high-risk patients with acute cholecystitis: a systematic review and pooled analysis. Surg Endosc 2016; 30: 5200-8.

32. Dollhopf M, Larghi A, Wil U, et al. EUS-guided gallbladder drainage in patients with acute cholecystitis and high surgical risk 
using an electrocautery-enhanced lumen-apposing metal stent device. Gastrointest Endosc 2017; 86: 636-43.

33. Teoh AY, Binmoeller KF, Lau JY. Single-step EUS-guided puncture and delivery of a lumen-apposing stent for gallbladder drainage using a novel cautery-tipped stent delivery system. Gastrointest Endosc 2014; 80: 1171.

34. Choi JH, Lee SS, Choi JH, et al. Long-term outcomes after endoscopic ultrasonography-guided gallbladder drainage for acute cholecystitis. Endoscopy 2014; 46: 656-61.

35. Takagi W, Ogura T, Sano T, et al. EUS-guided cholecystoduodenostomy for acute cholecystitis with an anti-stent migration and anti-food impaction system: a pilot study. Therap Adv Gastroenterol 2016; 9: 19-25.

36. Walter D, Teoh AY, Itoi T, et al. EUS-guided gall bladder drainage with a lumen-apposing metal stent: a prospective long-term evaluation. Gut 2016; 65: 6-8.

Received: 23.08.2018, accepted: 10.10.2018. 